\title{
A Prelude to the Fractional Calculus Applied to Tumor Dynamic*
}

\author{
N. VARALTA ${ }^{1}$, A.V. GOMES ${ }^{1}$ and R.F. CAMARGO ${ }^{2 * *}$
}

Received on December 9, 2013 / Accepted on June 14, 2014

\begin{abstract}
In order to refine the solution given by the classical logistic equation and extend its range of applications in the study of tumor dynamics, we propose and solve a generalization of this equation, using the so-called Fractional Calculus, i.e., we replace the ordinary derivative of order 1, in one version of the usual equation, by a non-integer derivative of order $0<\alpha \leq 1$, and recover the classical solution as a particular case. Finally, we analyze the applicability of this model to describe the growth of cancer tumors.
\end{abstract}

Keywords: biomathematics, fractional calculus, logistics equation, dynamics of cancer tumor.

\section{INTRODUCTION}

The art of getting a differential equation whose solution describes well the reality, brings a great difficulty, generally the closer we are to perfectly describe a real problem the harder and complex the equations involved are, in Albert Einstein words [2] "One thing I have learned in a long life: all our science, measured against reality, is primitive and childlike - and yet it is the most precious thing we have".

In this sense, the so-called Fractional Calculus, which is the branch of mathematics that deals with the study of integrals and derivatives of non-integer orders, plays an outstanding role. There are countless problems that, when described in terms of differential equations of non integer order, provide a more accurate description of reality. Specially phenomena that posses time dependence, since the fractional derivatives excellently describe effects of memory and hereditary $\operatorname{properties}^{3}[2,19]$.

Among the different ways to solve a fractional differential equation (FDE), or still an initial value problem, we mention the so-called method of integral transforms, i.e., we transform the

\footnotetext{
*Paper presented at CMAC-SE.

**Corresponding author: Rubens de Figueiredo Camargo.

${ }^{1}$ Department of Biostatistics, IBB, UNESP - Universidade Estadual Paulista, 18618-970 Botucatu, SP, Brazil.

E-mails: najla@ibb.unesp.br; ariannevellasco@gmail.com

2 Department of Mathematics, FC, UNESP - Universidade Estadual Paulista, 17033-360 Bauru, SP, Brazil.

E-mail: rubens@fc.unesp.br

${ }^{3}$ Indeed, fractional derivative is not a local operator, since it is defined in terms of the fractional integral, as it will be seen in equation (2.10).
} 
initial problem into another one (called transformed problem and normally easier to be solved), solve this new problem and recover the original solution applying the inverse integral transform $[2,3,19]$. Usually the solution of a FDE is expressed in terms of a parameter corresponding to the order of the derivative, and the solution of the corresponding integer order equation is recovered for a particular value of this parameter. In many cases, it is not a whole order of the derivative that makes the solution of equation closer to reality $[2,4,5]$.

Moreover, the logistic equation was published in 1838 by Pierre François Verhulst to model the growth of world population and was based on the assessment of population statistics available, complementing the theory of exponential growth of Thomas Robert Malthus. It can be applied to models with time dependence and has a wide application area since the inhibiting factors are taken into consideration. Furthermore, the logistic equation proved applicable in a series of probabilistic events and related to chaos theory and industrial and business dynamics [7, 25].

Recently, the logistic equation has been applied to describe the growth of populations both in the laboratory natural habitat, limiting the growth by influence of competition, mortality and fertility factors. However, the logistic model does not fit very well in cases where there are more complex relationships acting as interactions within food webs or several features that occur in most cases in nature dependencies[5, 6, 7, 8, 9, 22]. Aiming to generalize the logistic equation and give a better description for some of those events, El-Sayed, at all [5], studied the corresponding fractional-order logistic by a numerical analyze.

The purpose of this paper is to present the analytic solution of the fractional logistic equation, in the inverse form, and analyze the applicability of this fractional equation to improve the description of the dynamic of cancer tumor and compare this model with some classical models presented in the literature [9, 18], and is organized as follows. In Section 1 we make a brief introduction to our work. In Section 2 we present some functions inherent to fractional calculus and the definition of the fractional integral of Riemann-Liouville and Caputo's fractional derivative. In Section 3 we present the Classical Logistic Equation and its corresponding solution and also its generalization via Fractional Calculus. Besides that, we recover the classical solution as a particular case. In Section 4 we present some classical models presented in literature that describe the growth of tumors and compare them with the solution of fractional logistic equation. Finally in Section 5 we present our concluding remarks.

\section{FRACTIONAL CALCULUS}

In this section we present the definition of some important special functions related to fractional calculus and their basic properties, that will allow us to propose and solve the fractional version of the classical logistic equation.

\subsection{Gamma Function}

We denote by $\Gamma(z)$ the Gamma function, this function can be defined by the improper integral [19]:

$$
\Gamma(z)=\int_{0}^{\infty} e^{-t} t^{z-1} d t,
$$


with $\operatorname{Re}(z)>0$, the integral is convergent.

Solving this integral by parts and a change of variable, it follows that:

$$
\Gamma(z+1)=z \Gamma(z) .
$$

Once that $\Gamma(1)=1$, it is easy to see that, for $n \in \mathbb{N}, \Gamma(n+1)=n$ !, i.e., the Gamma function is a generalization of factorial ${ }^{4}$.

\subsection{Gel'fand-Shilov function definition}

Let $n$ and $v$ be, respectively, a natural and non-integer number with $\operatorname{Re}(v)>0$, we define the Gel'fand-Shilov function as

$$
\phi_{n}(t)=\left\{\begin{array}{cc}
\frac{t^{n-1}}{(n-1) !} & \text { se } t \geq 0 \\
0 & \text { se } t<0 .
\end{array} \quad \text { and } \quad \phi_{\nu}(t)=\left\{\begin{array}{cc}
\frac{t^{\nu-1}}{\Gamma(\nu)} & \text { se } t \geq 0 \\
0 & \text { se } t<0 .
\end{array}\right.\right.
$$

Thus, the Laplace transform of $\phi_{v}(t)$ is given by:

$$
\mathfrak{Q}\left[\phi_{\nu}(t)\right]=\int_{0}^{\infty} e^{-s t} \frac{t^{\nu-1}}{\Gamma(\nu)} d t=\frac{1}{\Gamma(v)} \int_{0}^{\infty} e^{-a}\left(\frac{a}{s}\right)^{\nu-1} \frac{d a}{s}=s^{-v},
$$

in which, the second equality is due to the change of variable $(s t=a)$ and the last equality is due to the definition of gamma function.

\subsection{Mittag-Leffler functions}

The classical Mittag-Leffler function is a complex function depending on a complex parameter and was defined and studied by Mittag-Lefller [14, 15, 16], in the year 1903, as

$$
E_{\alpha}(z)=\sum_{k=0}^{\infty} \frac{z^{k}}{\Gamma(\alpha k+1)}, \quad \operatorname{Re}(\alpha)>0 .
$$

This function is a direct generalization of the exponential function since for $\alpha=1$ we have the exponential function. The function defined by

$$
E_{\alpha, \beta}(z)=\sum_{k=0}^{\infty} \frac{z^{k}}{\Gamma(\alpha k+\beta)} \quad \operatorname{Re}(\alpha), \operatorname{Re}(\beta)>0,
$$

gives a generalization of $E_{\alpha}(z)$ since $E_{\alpha, 1}(z)=E_{\alpha}(z)$. This generalization was studied by Wiman [26] in 1905.

The Laplace transform of the function $t^{\beta-1} E_{\alpha, \beta}\left(a t^{\alpha}\right)$, is given by:

$$
\mathfrak{L}\left[t^{\beta-1} E_{\alpha, \beta}\left( \pm a t^{\alpha}\right)\right]=\frac{s^{\alpha-\beta}}{s^{\alpha} \mp a} \text {. }
$$

\footnotetext{
${ }^{4}$ As we will see, the generalization of factorial, by the gamma function, is the key for the generalization of the usual calculus by the fractional calculus.
} 
One important relation between the Mittag-Lffler functions with one and two parameters is given by

$$
E_{\alpha, \alpha+1}(z)=\sum_{n=0}^{\infty} \frac{(z)^{n}}{\Gamma(\alpha n+\alpha+1)}=\frac{1}{z} \sum_{n=0}^{\infty} \frac{(z)^{n+1}}{\Gamma[\alpha(n+1)+1]}=\frac{1}{z}\left[E_{\alpha}(z)-1\right]
$$

\subsection{Fractional integral}

Here we first define the integral operator of order $n$ and then, using the generalization of factorial by Gamma function, we define the fractional integral of Riemann-Liouville.

Definition. Let $n \in \mathbb{N}$ and $f(t): \mathbb{R} \rightarrow \mathbb{R}$ an integrable function. We define the Integral Operator, $I$, of order 1 and $n$ respectively as:

$$
I f(t)=\int_{0}^{t} f\left(t_{1}\right) \mathrm{d} t_{1} \quad \text { and } \quad I^{n} f(t)=\int_{0}^{t} \int_{0}^{t_{1}} \ldots \int_{0}^{t_{n-1}} f\left(t_{n}\right) \mathrm{d} t_{n} \mathrm{~d} t_{n-1} \ldots \mathrm{d} t_{1} .
$$

Theorem. For $f(t): \mathbb{R} \rightarrow \mathbb{R}$ integrable, we have that [2]:

$$
I^{n} f(t)=\phi_{n}(t) * f(t)=\int_{0}^{t} \phi_{n}(t-\tau) f(\tau) d \tau=\int_{0}^{t} \frac{(t-\tau)^{n-1}}{(n-1) !} f(\tau) d \tau,
$$

where * denotes the Laplace convolution product and $\phi_{n}(t)$ is the Gel'fand-Shilov function.

Definition. Let $f(t)$ be an integrable function and $v \in \mathbb{C}$, such that Re $(v)>0$, the RiemannLiouville fractional integral of order $v$ of $f(t)$, denoted by $I^{v} f(t)$, is defined as ${ }^{5}$ :

$$
I^{v} f(t)=\phi_{\nu}(t) * f(t)=\int_{0}^{t} \frac{(t-\tau)^{\nu-1}}{\Gamma(\nu)} f(\tau) \mathrm{d} \tau
$$

\subsection{Caputo's Fractional Derivative}

Caputo's fractional derivative is based on the fact that derivative is the left inverse operator of integral and on Lagrange's exponential law.

According to Caputo, the fractional derivative is the fractional integral of an integer order derivative such that exponential law makes sense [10].

Thus, let $f(t)$ be a differentiable function, $m \in \mathbb{N}$ and $\alpha \notin \mathbb{N}$ such that $m-1<\operatorname{Re}(\alpha)<m$, we have:

$$
D^{\alpha} f(t)=I^{m-\alpha} D^{m} f(t)=\phi_{m-\alpha}(t) * D^{m} f(t)
$$

\footnotetext{
${ }^{5}$ Note that this definition comes from equation (2.8) and from the fact that Gel'fand-Shillov function is well defined for non integer numbers.
} 


\subsection{Laplace Transform}

From the definition (2.10) and from the Laplace convolution theorem we may write:

$$
\mathfrak{L}\left[D^{\alpha} f(t)\right]=\mathfrak{Q}\left[\Phi_{m-\alpha}(t)\right] \mathfrak{L}\left[D^{m} f(t)\right]=s^{\alpha-m} \mathfrak{Q}\left[D^{m} f(t)\right] .
$$

\section{FRACTIONAL LOGISTIC EQUATION}

In this section, we present the classic logistic equation and its solution and also their respective generalized using fractional calculus.

\subsection{The Classic Logistic Equation}

In 1838, Verhulst [25] published logistic equation defined as:

$$
\frac{d}{d t} N(t)=k N(t)\left(1-\frac{N(t)}{r}\right)
$$

where $N(t)$ is the number of individuals in time $t, k$ is the intrinsic growth rate and $r$ is carrying capacity. Without loss of generality, taking $r=1$, this equation can be rewritten in the form:

$$
\frac{d}{d t} N(t)=k N(t)[1-N(t)]
$$

Note that this is a non-linear and separable equation. Though solving this equation is quite simple we cannot say the same about its fractional version. Using the variable change $v(t)=1 / N(t)$ in the previous equation we obtain the following Linear and separable equation:

$$
\frac{d v(t)}{d t}=k[1-v(t)]
$$

whose solution is given by

$$
v(t)=1+\frac{1}{c} \mathrm{e}^{-k t} \Rightarrow v(0)=1+\frac{1}{c} .
$$

Since $N(t)=v(t)^{-1}$, we can write $1 / c=1 / N(0)-1$, as a result;

$$
N=\frac{1}{e^{-k t} c^{-1}+1} \Rightarrow N=\frac{1}{1+\left[\frac{1}{N(0)}-1\right] e^{-k t}} .
$$

Note that $0<N(0)<1$ and $\lim _{t \rightarrow \infty} N(t)=1$ (i.e. the carrying capacity).

\subsection{Fractional Logistic Equation (Inverse form)}

We will use the results presented above to propose a generalization, via fractional calculus, for the logistic equation in the linear form given by equation (3.3). It is very important to note that the fractional version with order $0<\alpha \leq 1$ of equation (3.1) was numerically solved by El-Sayed, at all [5], however, we will present the analytic solution of equation (3.3). 
The fractional version of equation (3.3) with order $0<\alpha \leq 1$ is given by

$$
\frac{d^{\alpha} v(t)}{d t^{\alpha}}=D^{\alpha} v(t)=k[1-v(t)] .
$$

Applying Laplace transform to both sides, following:

$$
\mathfrak{\Omega}\left[D^{\alpha} v(t)\right]=k \Omega[1-v(t)] .
$$

Using equation (2.11), with $m=1$ (since $0<\alpha \leq 1$ ) we have:

$$
s^{\alpha} F(s)-s^{\alpha-1} v(0)=k\left[\frac{1}{s}-F(s)\right]
$$

Then

$$
F(s)=k\left[\frac{s^{-1}}{s^{\alpha}+k}\right]+v(0)\left[\frac{s^{\alpha-1}}{s^{\alpha}+k}\right],
$$

in which $F(s)=\mathfrak{2}[v(t)]$.

Thus we have:

$$
v(t)=\mathfrak{Q}^{-1} \mathfrak{Q}[v(t)]=k \mathfrak{Q}^{-1}\left[\frac{s^{-1}}{s^{\alpha}+k}\right]+v(0) \mathbb{R}^{-1}\left[\frac{s^{\alpha-1}}{s^{\alpha}+k}\right] .
$$

From this, the result of equation (2.6), we have:

$$
v(t)=t^{\alpha} k E_{\alpha, \alpha+1}\left(-k t^{\alpha}\right)+v(0) E_{\alpha}\left(-k t^{\alpha}\right) .
$$

Then, using equation (2.7) with $z=-k t^{\alpha}$

$$
v(t)=1+E_{\alpha}\left(-k t^{\alpha}\right)[v(0)-1] .
$$

Once we $v(t)=1 / N(t)$, we obtain:

$$
N(t)=\frac{1}{1+\left[\frac{1}{N(0)}-1\right] E_{\alpha}\left(-k t^{\alpha}\right)} .
$$

Note that, $\lim _{\alpha \rightarrow 1} N(t)=\frac{1}{1+\left[\frac{1}{N(0)}-1\right] e^{-k t}}$, i.e., the solution of integer order is a particular case of the fractional solution.

Below is the graph corresponding to the solution of equation (3.5), taking $N(0)=0.2$ carrying capacity $r=1$, for different values of $\alpha$, we have:

Since $\lim _{t \rightarrow \infty} E_{\alpha}\left(-k t^{\alpha}\right)=0$ for all values $0<\alpha \leq 1$ we have:

$$
\lim _{t \rightarrow \infty} N(t)=\lim _{t \rightarrow \infty} \frac{1}{1+\left[\frac{1}{N(0)}-1\right] E_{\alpha}\left(-k t^{\alpha}\right)}=1,
$$

that is, the all the values considered converge to the value of the carrying capacity. 


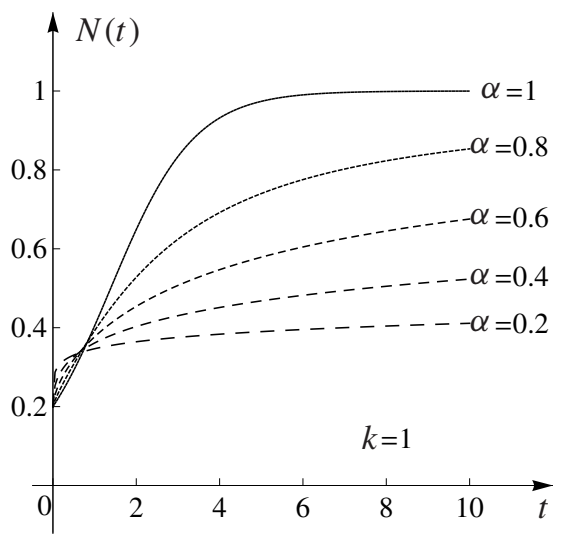

Figure 1: The solution of equation (3.5).

\section{TUMOR DYNAMIC}

With the artifice of scientific research and the use of mathematics in modeling, description and even the prediction of a given physical process we can better understand some factors in the biological context. However, such use is recent, particularly in the study of cancer tumors one of the reasons is that we still finding difficulty in modeling satisfactorily the tumor's growth. The models found are currently based on computer simulations, differential equations and curve fitting, but even the most sophisticated models are unable to predict how the size of the host interferes in tumor's growth. There are several models of tumor growth in the literature, thus, it is very important to know which one best fits with experimental data. It is of paramount importance to understand the assumptions and the consequences of such models because often these models supports more complex models of tumor growth [5, 6, 7, 8, 9, 22].

It can be considered a volume of tissue as a "cellular community" comprises "species" of mesenchymal and epithelial cells in dynamic equilibrium with the environment and with each other [8]. A small number of tumor cells produced by successive non-lethal mutations begin to interact with the community of normal cells and do not recognize, thus triggering the acquisition of space and the vital resources of the existing community.

In the case of tumor dynamic saturation in the growth of various types of tumors is not well modeled by the exponential model. For this reason, this model applies only to avascular tumors, i.e., when angiogenesis has not occurred, which under Kerbel [11], have around 1 to $2 \mathrm{~mm}$ diameter [21, 22].

Indeed, tumor cells compete for oxygen and vital resources that is the reason why the logistic model fits well in several cases[12, 25]. In the literature, one realizes that tumor growth does not follow a universal law [20], so we can mention another two of the most used models ${ }^{6}$ :

\footnotetext{
${ }^{6}$ There are several other models that describe the dynamic of cancer tumor that will not be considered here [8], due to the fact that they are very similar to those that we considered.
} 
i) The so called Generalized Logistic model is given by [8]

$$
\frac{d N(t)}{d t}=\frac{r}{\theta} N(t)\left(1-\frac{N(t)}{k}\right)^{\theta}
$$

This model describes well the growth of breast cancer [23].

ii) The Gompertz model [8],

$$
\frac{d N(t)}{d t}=-r N(t) \ln \left(\frac{N(t)}{k}\right),
$$

best fits the volumetric growth in vivo [13];

Below, we present some graphs of models in the literature that describe the increase in tumor mass/volume over time, from the simple Exponential growth model from one parameter to the more sophisticated models such as Gompertz, Generalized Logistic and Fractional Logistic Equation.

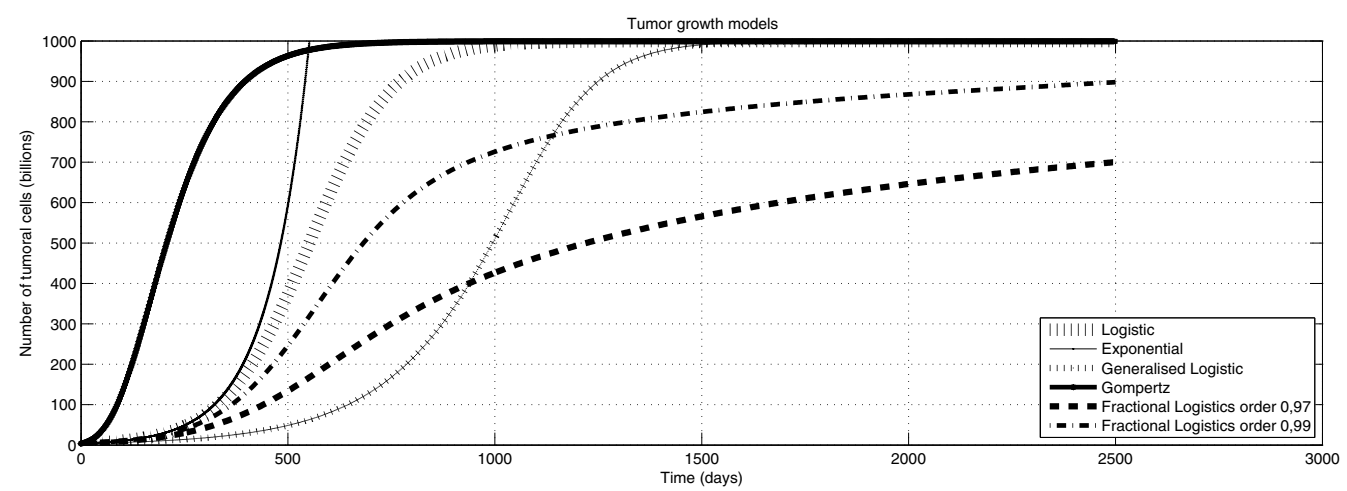

Figure 2: Tumor growth in humans according to the Exponential Models, Logistic, Gompertz and Logistic Fractional.

In the chart above tumor growth in humans we consider the initial population $N(0)=4 \times 10^{9}$, a carrying capacity cells $r=10^{12}, k=10^{-2} /$ day and for the generalized logistic model $\theta=2$ with scale $1: 1.000 .000 .000$.

One disadvantage of all the usual models is that they go to the carrying support faster than what is expected $[5,6,7,8,9,22]$. This is one of the advantages of the fractional logistic model, i.e., we note that as the order of the derivative decreases the amount of convergence to the support becomes slower. This slow convergence to the carrying support is consistent with the growth of some types of cancer tumor [8] making this highly relevant for the study of tumor dynamic equation, since this model include competition between tumor cells for vital resources and predicts that the maximum size of a tumor takes longer to be achieved. 


\section{CONCLUDING REMARKS AND FUTURE WORK}

The Fractional Calculus is nearly as old as the entire order one, but just in end of last century this valuable tool became evident, especially in modeling phenomena that possess time dependence, since fractional derivatives excellently describe memory effects when encountered derived from their entire order.

In this work, we had the purpose of emphasizing the importance of Fractional Calculus to generalize and refine the solution of differential equations of integer order, these being of paramount importance in describing various phenomena in biological field. With this, we propose a generalization via Fractional Calculus for classical logistic equation and show that the fractional model may provides a better description than the usual on, in the study of cancer tumor growth.

One important aspect is to analyze if the solutions of the fractional versions of equations (3.1) and (3.3) are the same. Despite having scientific value the search for an answer to the previous question, is not indispensable for the purpose of the article, since equations (3.1) and (3.3) are equivalents, to consider the fractional growth we may to choose one of them. We are working on an answer for this question and we intend to publish it in a future work.

A natural continuation of this work is to study the system presented by Rodrigues [21, 22] in fractional version, since we know the behavior of tumor free from chemotherapy we will use this knowledge to analyze the increase or decrease in tumor cancer on the action of chemotherapeutic agents.

RESUMO. Com o intuito de refinar a solução da clássica equação logística e ampliar seu campo de aplicação para o estudo da dinâmica tumoral, propomos e resolvemos a generalização fracionária desta equação, utilizando o assim chamado cálculo fracionário, i.e., substituímos a derivada ordinária da equação usual por uma derivada fracionária de ordem $0<\alpha \leq 1$, e recuperamos a solução clássica como um caso particular. Por fim, analisamos a aplicabilidade deste modelo para descrever o crescimento de tumores de câncer.

Palavras-chave: biomatemática, cálculo fracionário, equação logística, dinâmica tumoral de câncer.

\section{REFERENCES}

[1] R.F. Camargo, E.C. Oliveira \& Ary O. Chiacchio. Teorema de Adição para as Funções de MittagLeffler. TEMA, 10(1) (2009), 1-8.

[2] R.F. Camargo. Cálculo Fracionário e Aplicações. Tese de Doutorado, IMECC, UNICAMP (2009).

[3] R.F. Camargo, Ary O. Chiacchio \& E. Capelas de Oliveira. Differentiation to Fractional Orders and the Fractional Telegraph Equation. J. Math. Phys., 49, 033505, (2008).

[4] R.F. Camargo, Ary O. Chiacchio \& E. Capelas de Oliveira. On anomalous diffusion and the fractional generalized Langevin equation for a harmonic oscillator. J. Math. Phys., 50, 123518, (2009). 
[5] A.M.A El-Sayed, A.E.M. El-Mesiry \& H.A.A El-Saka. One the fractional-order logistic equation. Applied Mathematics Letters, 20 (2007), 817-823.

[6] G.H. Erjaee, M. Shahbazi \& A. Erjaee. Dynamical analysis of mathematical model presented by fractional differential equations, describing the interaction between leukemic cancer cells, $\mathrm{T}$ cells and drug treatment with a drug optimal control. Open Access Scientific Reports, 1 (2012), 1-8.

[7] U. Forys \& A. Marciniak-Czochra. Logistic equations in tumour growth modelling. International Journal of Applied Mathematics and Computer Science, 13 (2003), 317-325.

[8] R.A. Gatenby \& T.L. Vincent. Application of quantitative models from population biology and evolutionary game theory to tumor therapeutic strategies. American Association for Cancer Research, 2 (2013), 919-927.

[9] P. Gerlee. The model muddle: in search of tumour growth laws. Cancer Research, 73 (2013), 24072411.

[10] R. Gorenflo \& F. Mainardi. Fractional Calculus: Integral and Differential Equations of Fractional Order. CISM Lectures Notes, (2000), 223-276.

[11] R.S. Kerbel. Tumor angiogenesis: past, present and the near future. Carcinogenesis, 21 (2000), 505515.

[12] A. Lotka. Meeting on the problem o forecasting city populations with special reference to New York city. Journal of the American Statistical Association, 20 (1925).

[13] S. Michelson, A.S. Glicksman \& J.T. Leith. Growth in solid heterogeneous human colon adenocarcinomas: comparison of simple logistical models. Cell Prolif, 20 (1987), 343-355.

[14] G.M. Mittag-Leffler. Une generalisation de 1. integrale de Laplace-Abel. Comptes Rendus de l'Academie des Sciences Serie II, 137 (1903), 537-539.

[15] G.M. Mittag-Leffler. Sur la nouvelle fonction $E_{\alpha}(x)$. Comptes Rendus de l'Academie des Sciences, 137 (1903), 554-558.

[16] G.M. Mittag-Leffler. Sur la representation analytiqie d'une fonction monogene (cinquieme note). Acta Mathematica, 29(1) (1905), 101-181.

[17] E.C. Oliveira. Funções Especiais com Aplicações. Editora Livrariada Física, São Paulo (2005).

[18] C. Phipps. Combination of Chemotherapy and Antiangiogenic Therapies: A Mathematical Modelling Approach. Thesis the Master Degree, University of Waterloo, Canada (2009).

[19] I. Podlubny. Fractional Differential Equation - An Introduction to Fractional Derivates, Fractional Differential Equations, to Methods os their Solution and some of their Applications. Academic Press, San Diego, 198 (1999).

[20] M. Retsky. Letter to the editor. J. Theor. Biol., 229 (2004), p. 289.

[21] D.S. Rodrigues. Modelagem matemática em câncer: dinâmica angiogência e quimioterapia antineoplásica. Dissertação de Mestrado, UNESP, IBB (2011).

[22] D.S. Rodrigues, P.F.A. Mancera \& S.T.R. Pinho. Modelagem Matemática em Câncer e quimioterapia: uma introdução. Notas em Matemática Aplicada, SBMAC, São Carlos - SP, Brasil, Volume 58, eISSN 2236-5915, (2011).

[23] J.S. Spratt, J.S. Meyer \& J.A. Spratt. Rates of growth of human neoplasms: part II. J. Surg. Oncol., 61 (1996), 68-73. 
[24] V.G. Vaidya \& F.J. Alexandro-Jr. Evaluation of some mathematical models for tumor growth. Int. J. Bio-med. Comp., 13 (1982), 19-35.

[25] P.F. Verhulst. Notice sur la loi que la population poursuit dans son accroissement. Correspondance mathématique et physique, 10 (1838), 113-121.

[26] A. Wiman. Über den fundamentalsatz in der theorie der funktionen $E_{\alpha}(z)$. Acta Math., 29 (1905), 191-201. 